\title{
Kinetic and Thermodynamic Study in Pozzolanic Chemical Systems as an Alternative for Chapelle Test
}

\author{
Marco César Prado Soares ${ }^{a} \oplus^{\oplus}$, Beatriz Ferreira Mendes ${ }^{a}$, Egont Alexandre Schenkel ${ }^{b}$, Murilo \\ Ferreira Santos ${ }^{b}$ Eric Fujiwara ${ }^{a}$, Carlos Kenichi Suzuki ${ }^{b}$
}

${ }^{a}$ Laboratório de Materiais e Dispositivos Fotônicos,Universidade de Campinas, Rua Mendeleyev, 200, Bloco
K, 13083-872, Campinas, SP, Brasil
${ }^{b}$ Departamento de Materiais e Engenharia de Manufatura, Universidade de Campinas, Campinas, SP, Brasil

Received: February 20, 2018; Revised: April 06, 2018; Accepted: May 11, 2018

Important properties in cementitious materials, such as concrete, are related to the presence of additives that influence the rigidity and the physical and chemical resistances. For the evaluation of the additive effectiveness, known as pozzolanic activity, a simple procedure, the Chapelle test, is commonly used, and it essentially consists in a reaction between the additive with calcium oxide in aqueous medium. However, such procedure presents limitations in terms of processing time and lack of information regarding the reactions kinetics. In this sense, a simple method based on the kinetic and thermodynamic principles of chemical reactions is proposed, which can be performed using conventional electronic $\mathrm{pH}$ sensors. The study provides an alternative methodology with many advantages over the traditional procedure, such as energy and time-savings, more robustness and more confidence. In this paper, three types of silica nanoparticles that can be used as low-cost additives were characterized in relation to their morphology and crystallinity by XRD and SEM, the particles average diameters were obtained and the particles were used for studying the chemical process that takes place during the Chapelle test. Results and the semi-empirical analysis provided strong evidence that the process is an acid-base 1:1 reaction and it was verified that the mean reaction times varied from 64 to $195 \mathrm{~min}$. It is a remarkable result, since the proposed analysis can be performed with simple, fast and low-cost instrumentation and needs only a worksheet software, whereas the Chapelle test takes 16 hours and provides no dynamic information. Besides the limitation that the methodology is not able to quantify and to elucidate the effects of the specific surface area of the particles, which needs a complete BET study, the research provides a significant contribution for the understanding of the pozzolanic process, of great importance in both concrete and ceramic research.

Keywords: Chapelle test, pozzolanic reaction, concrete, silica, quartz.

\section{Introduction}

The advances in concrete technology rely on the use of mineral admixtures, such as silica fume, in order to produce high-performance products ${ }^{1}$. The utilization of those materials enhances the concrete properties, including durability, resistance to chloride corrosion, freezing, thawing, and deicing salting scale ${ }^{2,3}$.

Additions constituted of siliceous or aluminous materials in finely divided form and generally in the presence of water are usually referred as "Pozzolana"4,5. These materials react chemically with calcium hydroxide and produce compounds with cementitious properties $\mathrm{s}^{4,5}$.

The effectiveness of these additions is termed pozzolanic activity, being commonly evaluated using the Chapelle tes ${ }^{4,6}$, a simple procedure in which $1 \mathrm{~g}$ of the analyzed sample is added to $200 \mathrm{~mL}$ of deionized water and $1 \mathrm{~g}$ of calcium oxide. The mixture is boiled for $16 \mathrm{~h}$, and then it is filtered and titrated with a $1 N$ chloride acid solution, with phenolphthalein indicator, in order to evaluate the amount of calcium oxide that has not taken part in the reaction ${ }^{5}$.
The Chapelle test is relatively simple, but it does not provide information about the dynamic behavior of the chemical process. A first problem that can be pointed out is that a $16 \mathrm{~h}$ time is sufficiently long for occurring parallel reactions, such as the acid-base neutralization reaction of the calcium oxide by the carbon dioxide present in the air $^{7}$, giving a false interpretation to the test result.

The test is also not able to quantify common effects in chemical reactions that can change the observed rate ${ }^{8}$, like agitation power, solid-liquid equilibrium effects ${ }^{9}$, reaction limitations by the increase of mass transfer resistance, and so on. Moreover, it does not provide important information about the intrinsic kinetics of the process, which can help to elucidate the mechanism of the reaction ${ }^{8}$.

It is also important to realize that the colorimetric indicators, like phenolphthalein, are not able to provide a specific value of $\mathrm{pH}$, since it has a characteristic range in which its color changes ${ }^{10}$. Important errors can be added to the analysis, depending on the color of the analyzed medium and depending on the difficulty of the analyst in 
differentiating the exact titration equivalence point ${ }^{10}$. Such errors are increased if the analyte is in low concentration, and this technique is particularly sensitive to the analyst experience in performing the procedure ${ }^{10}$.

In this sense, the objective of the present work is to analyze the kinetics of the pozzolanic reaction process, with three different silica nanoparticles reacting with calcium oxide in aqueous solutions. These materials present relative low-cost, what makes them economically attractive for the use as concrete additives. The studied samples were characterized and chemically tested, providing information about their morphology and about the kinetics of the reactions involved.

In order to help the elucidation of the reaction and to correct the temperature effects on the water ionization chemical equilibrium - the chemical equilibrium constant can reach values one order of magnitude higher than the value for $25^{\circ} \mathrm{C}\left(10^{-14}\right)$ for temperatures lower than $100^{\circ} \mathrm{C}^{9}-$ the principles of the chemical equilibrium thermodynamics and the chemical kinetics are applied to the analysis. A particular mechanism is hypothesized, and it is shown that the test can be performed at lower temperatures and during less time than the ones used for Chapelle test, providing important energy savings and faster analysis. The use of an electronic, simple and non-expensive device - the $\mathrm{pH}$ meter - also provides more accuracy and confidence to the results obtained, consisting in a useful and fast methodology for the evaluation of the effectiveness of the pozzolanic addition in concrete.

Due to the wide use of concrete, silica, and other ceramic materials on the urban societies, it is of great importance to understand the intrinsic mechanisms that take place when they interact, making it possible to optimize the processes and to find alternatives friendlier in both the economic and environmental perspectives.

\section{Materials and Methods}

\subsection{Thermodynamic analysis of the aqueous solution}

In order to evaluate the dynamics of the pozzolanic reaction, the thermodynamic analysis of the aqueous medium must be analyzed properly and the corrections on $\mathrm{pH}$ scale with temperature must be performed. In the absence of such rigorous treatment, the simple reading of the $\mathrm{pH}$ meter can lead to results of hydroxide ions concentration one or two orders of magnitude lower than the actual values. For example, a reading of $\mathrm{pH}$ equals to 11.2 corresponds to a concentration of $\mathrm{OH}^{-}$ions of $0.0016 \mathrm{~mol} / \mathrm{L}$ when the temperature is $25^{\circ} \mathrm{C}$, but the value for the temperature of 75 ${ }^{\circ} \mathrm{C}$, used in this research, is approximately equals to 0.036 $\mathrm{mol} / \mathrm{L}$, more than 22 times higher.

For correcting the scales, the authors recommend a construction of a worksheet in a software like Microsoft Excel, with a column for the $\mathrm{pH}$ meter readings, a column for the experiment temperature, and a column for each one of the equations listed in the text. The equations constitute an algorithm that should be solved in the indicated order, and it is possible to build a computer routine in Excel VBA for solving the more complex operations, with the any adequate numerical method, such as Newton-Rhapson or even a try-and-error procedure and the equations variables are summarized in Table 1. The integral calculus can be performed using the trapezoidal rule, and all the parameters needed are given in the References. The authors recommend that the parameters are put in fixed cells, and the use of the sheet is not restricted to the pozzolanic analysis - it can be used for the temperature correction of the $\mathrm{pH}$ reading in any aqueous medium.

Table 1. Nomenclature adopted.

\begin{tabular}{|c|c|}
\hline Variable & Meaning \\
\hline K & Chemical equilibrium constant \\
\hline$a_{i}$ & Chemical activity of species " $i$ " \\
\hline$v_{i}$ & Stoichiometric coefficient of species " $i$ " \\
\hline$\Delta G$ & Gibbs energy variation \\
\hline$R$ & Universal gases constant \\
\hline$T$ & Temperature \\
\hline$x_{i}$ & Molar fraction of species " $i$ " \\
\hline$\gamma_{i}$ & Activity coefficient of species " $i$ " \\
\hline$C_{p}$ & Specific heat \\
\hline$\Delta H$ & Enthalpy variation \\
\hline$\alpha$ & Debye-Hückel proportionality coefficient \\
\hline$z_{i}$ & Ionic charge of species " $i$ " \\
\hline$I$ & Ionic strength of reaction medium \\
\hline$m_{i}$ & Molality of species " $i$ " \\
\hline$\beta_{i k}$ & Interaction coefficient between species " $i$ " and " $k$ " \\
\hline$\rho$ & Solvent density at temperature " $T$ " \\
\hline$\varepsilon_{T}$ & Solvent dielectric constant at temperature " $T$ " \\
\hline$C_{A}$ & Molar concentration of the chemical species " $A$ " \\
\hline$C_{A 0}$ & $\begin{array}{l}\text { Initial molar concentration of the chemical species } \\
\text { " } A \text { " }\end{array}$ \\
\hline$k$ & Constant of reaction rate \\
\hline$t$ & Time of reaction \\
\hline
\end{tabular}

One of the reactants is the calcium oxide, which is known to elevate the water $\mathrm{pH}$ due to the liberation of hydroxide ions, accordingly to Equation $1^{7}$. The variation in $\mathrm{OH}^{-}$ions is then equals to the variation in $\mathrm{CaO}$ dissolved in water, and the $\mathrm{CaO}$ instant concentration can be calculated by interpreting the reading of a common electronic $\mathrm{pH}$ sensor, which gives the medium $\mathrm{pH}$ value when its probe is immersed in the aqueous suspension.

$$
\mathrm{CaO}_{(s)}+\mathrm{H}_{2} \mathrm{O}_{(l)} \rightleftarrows \mathrm{Ca}^{2+}{ }_{(a q)}+2(\mathrm{OH})_{(a q)}^{-}
$$


The water chemical equilibrium, expressed in Equation $2^{7}$, is disturbed by the presence of the hydroxide ions formed, and the equilibrium equation relates the concentration of acid ions, detected by the $\mathrm{pH}$ meter, to the concentration of hydroxide ions.

$$
\mathrm{H}_{2} \mathrm{O}_{(l)} \leftrightarrows{H^{+}}_{(a q)}+\mathrm{OH}_{(a q)}^{-}
$$

The chemical equilibrium is described by the equilibrium constant, $K$, which correlates the chemical activities of the species in the medium, according to Equation $3^{9}$.

$$
\mathrm{K}=\frac{a_{1} a_{2}}{a_{3}}=\prod_{i} a_{i}^{v i}
$$

In Equation 3, the indexes 1, 2 and 3 represent ion $\mathrm{H}^{(+)}$, ion $\mathrm{OH}^{(-)}$, and molecule $\mathrm{H}_{2} \mathrm{O}$, respectively. The equilibrium constant is related to the reaction Gibbs energy by Equation 4, and the activity of each specie is related to its molar fraction in solution by Equation $5^{9}$.

$$
\begin{gathered}
\Delta G(T)=-R T \ln K \\
a_{i}=x_{i} \gamma_{i}
\end{gathered}
$$

In a defined temperature $T$, it can be demonstrated from the classic van't Hoff equation that the variation in reaction enthalpy is given by Equation $6^{9}$. In this equation, $\Delta C_{p}$ is the sum of each reactant and product specific heat multiplied by the respective stoichiometric coefficient, and $T_{0}$ is a reference and arbitrary temperature.

$$
\int_{T_{0}}^{T} \Delta C_{p}(T)=\Delta H(T)
$$

The overall variation in reaction Gibbs energy, in a defined temperature, can be predicted from the variations in reaction enthalpy and in the reactants specific heat by Equation 7 . This equation is derived from Equation 6, combined to the Kirchhoff and Gibbs equations ${ }^{9}$, where $\Delta G^{0}$ and $\Delta H^{0}$ are the reaction Gibbs energy variation and enthalpy variation calculated in the reference temperature $T_{0}$, respectively.

$$
\frac{\Delta G(T)}{R T}=\frac{\Delta G^{0}-\Delta H^{0}}{R T_{0}}+\frac{\Delta H^{0}}{R T}-\frac{1}{R} \int_{T_{0}}^{T} \frac{\Delta H(T)}{T^{2}} d T
$$

The values of $\Delta H / T$ are well known for the ionization equilibrium of water and are available for a wide range of temperatures ${ }^{11}$, allowing the calculation of the reaction $\Delta G$ with Equation 7. Then, the equilibrium constant can be calculated by applying this result to Equation 4 .

As the water is always in large excess, with molar fractions above 0.9 , its activity can be predicted by the Lewis-Randall rule $^{12}$, and it is equal to the water molar fraction:

$$
a_{3}=x_{3}
$$

The activity coefficient of ionic species can be evaluated using the Debye-Hückel model for excess Gibbs energy ${ }^{13}$, which is expressed by Equation 9 .

$$
\ln \gamma_{i}=-\frac{\alpha\left(z_{i}\right)^{2} \sqrt{I}}{1+\sqrt{I}}+2 \sum_{k \neq 3} \beta_{i k} m_{k}
$$

The ionic strength, $I$, is defined by Equation 10. In the water system, the ionic charges are $z_{1}=+1$ and $z_{2}=-1$. Edwards et al. ${ }^{13}$ recommend the use of the values $\beta_{12}=\beta_{21}$ $=0.25 \mathrm{~kg} / \mathrm{mol}$ for the interaction between ions hydrogen and hydroxide.

$$
I \equiv \frac{1}{2} \sum_{j=1}^{3}\left(z_{j}\right)^{2} m_{j}
$$

In order to evaluate the Debye-Hückel coefficient $\alpha$, semi-empirical correlations can be applied, such as Equation $11^{14}$. Water density and water dielectric constant are also known for a wide range of temperatures ${ }^{15,16}$.

$$
\alpha=\frac{(1.824829238) \rho^{1 / 2}}{\left(\varepsilon_{T}\right)^{3 / 2}} \cdot 10^{6}
$$

After calculating the chemical activities in the reaction temperature, the species concentration can be evaluated by the application of the activity values in Equation 12, which is derived from Equations 3, 5 and 8 .

$$
K=\frac{a_{H^{+}} a_{O H}}{a_{H_{2} O}}=\frac{\left(x_{1} \gamma_{1}\right) \cdot\left(x_{2} \gamma_{2}\right)}{x_{3}}
$$

It is important to note that, defining $\mathrm{pH}$ and $\mathrm{pOH}$ according to Equations 13 and $14^{10}$, Equation 12 is simplified to the well-known Equation $15^{10}$ when the reaction proceeds under $25^{\circ} \mathrm{C}$.

$$
\begin{gathered}
p H=-\log \left(C_{H^{+}}\right) \\
p O H=-\log \left[C_{(O H)^{-}}\right] \\
p H+p O H=14
\end{gathered}
$$

\subsection{Reaction experiments}

In order to evaluate the kinetic behavior of a chemical reaction, it is necessary to analyze and to monitor a physical or chemical parameter which is related to the concentration of the species that are reacting ${ }^{8}$. Examples of such properties comprise the medium refractive index ${ }^{8}, \mathrm{pH}^{8}$, or viscosity ${ }^{17}$.

In this research, reaction experiments were conducted in a bench-scale batch reactor made of glass, with magnetic stirring and external temperature control, and the mixture volume was fixed in $250 \mathrm{~mL}$. The reactions were analyzed with a $\mathrm{pH}$ meter Gehaka PG 2000 able to measure $\mathrm{pH}$ and the sample temperature, equipped with electrode SC26 Sensoglass, adapted 
for handling temperatures up to $90{ }^{\circ} \mathrm{C}$ (Gehaka, Brazil). The reactions were monitored until no more variation in $\mathrm{pH}$ could be observed. The medium was kept at constant temperature, but each experiment was carried out in a different condition, with temperatures varying from 55 to $80{ }^{\circ} \mathrm{C}$.

Three different sources of silica were used: amorphous silica nanoparticles ("soot"), microcrystalline quartz, and amorphous commercial "active silica" (Tecnosil, Brazil). These particles were characterized by scanning electron microscopy (SEM) and by X-Ray diffraction (XRD).

The $\mathrm{pH}$ values measured by the equipment were converted to hydrogen concentration by applying Equation 13, and the thermodynamic Equations 3 to 12 were applied for the conversion of these data in hydroxide ions concentration. The instant hydroxide concentration is related to the instant calcium oxide concentration by Equation 1, since $\mathrm{CaO}$ is the main responsible for the medium alkalinity.

\subsection{Nanoparticles preparation}

Soot particles were synthesized by the Vapor Axial Deposition (VAD) method, which has been widely studied for the production of optical fiber preforms ${ }^{18}$. The silica particles produced by this methodology are completely amorphous and present spherical morphology ${ }^{18}$. The particles morphologies can be precisely controlled by adjusting the gases flow rates, such as $\mathrm{H}_{2}, \mathrm{O}_{2}, \mathrm{~N}_{2}$, and $\mathrm{SiCl}_{4}$ flow rates, and by controlling the burner-target distance $D$, the burnertarget inclination angle $\theta$, and the target vertical translation speed $\mathrm{v}^{18,19}$, as shown in Figure 1.

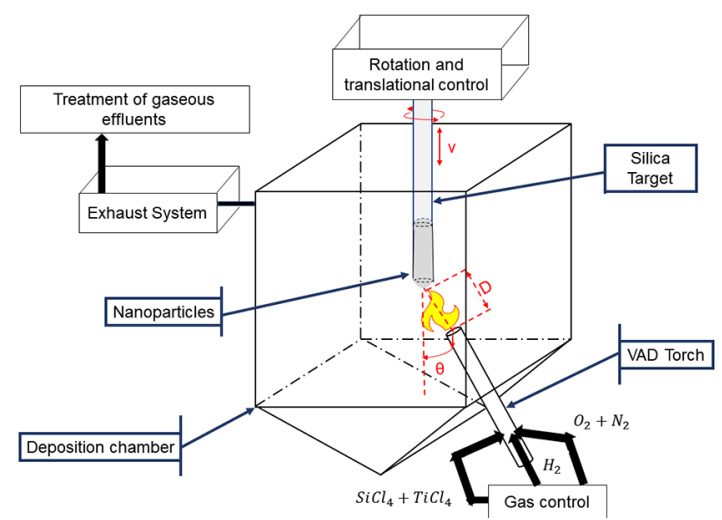

Figure 1. Scheme of the VAD chamber used for the synthesis of soot nanoparticles.

A high-temperature $\mathrm{O}_{2}-\mathrm{H}_{2}$ flame promotes the hydrolysis and oxidation of $\mathrm{SiCl}_{4}$, according to reaction 16 . The nanoparticles are axially deposited on the surface of a silica rod target in constant rotation and controlled vertical translation movements ${ }^{19}$.

$$
\mathrm{SiCl}_{4}+2 \mathrm{H}_{2}+\mathrm{O}_{2} \rightarrow \mathrm{SiO}_{2}+4 \mathrm{HCl}
$$

After the deposition, a "green body" is formed over the silica rod, as shown in Figure 2. This green body is mechanically separated from the rod and crushed in a silica mortar in order to separate the agglomerated nanoparticles, forming the silica powder (soot).

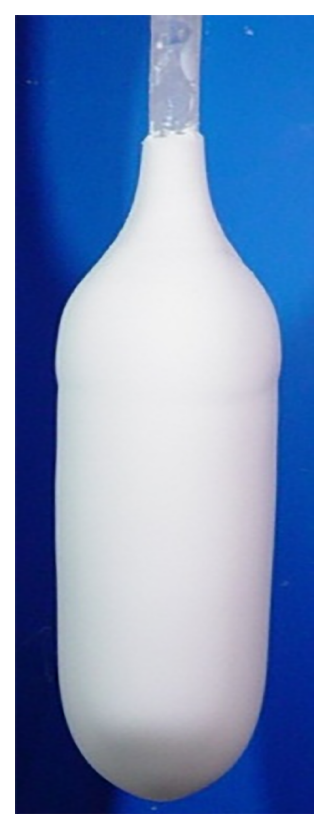

Figure 2. Green body formed by agglomerated silica nanoparticles synthesized by VAD.

The crystalline quartz microparticles were produced in-house using ball milling in silica medium, and quartz lumps were used as balls, in order to minimize the material contamination $^{20}$. The mill is loaded with the quartz sand and deionized water and is subjected to constant rotation (fill factor $\mathrm{K}=0.42)^{21}$. The quartz balls comminute the sand and progressively reduce the crystal dimensions. The milling time was fixed in $165 \mathrm{~h}$, and the rotation speed was set to $100 \mathrm{rpm}^{22}$.

After the milling process, the mixture of milled quartz and water was transferred to a $2 \mathrm{~L}$ graduated cylinder and completed with deionized water. After $48 \mathrm{~h}$, the large particles were sedimented, whereas submicron particles remained floating in the translucid phase, as shown in Figure 3. Then, this clarified portion was separated and the water was evaporated, forming a fine powder, yielding micro and nanostructured crystals.

\subsection{Mechanistic hypothesis and integral analysis}

Since in standard temperature and pressure conditions (STP) silica can be classified as an acid oxide, weakly ionizable in water, getting a negative charge ${ }^{7}$, and calcium oxide is a basic oxide, as shown in Equation 1, it can be hypothesized that the pozzolanic reaction consists in a LuxFlood acid-base reaction ${ }^{23}$. 


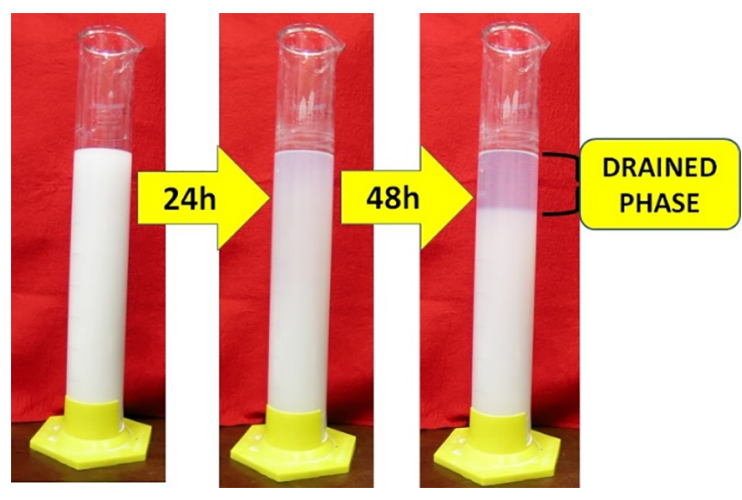

Figure 3. Decanting process. After $48 \mathrm{~h}$, the translucent phase is drained and the water is evaporated, forming a powder containing submicron particles.

Lux-Flood concept defines acid as a species which is able to receive oxide ions, whereas a base is a species which is able to donate oxide ions ${ }^{23}$. Lux-Flood definition is mainly applied to fused oxide and fused salt systems and can be understood as the neutralization of an acid oxide by a basic oxide ${ }^{7,23}$.

It is important to realize that, in aqueous solutions, the Lewis acid-base definition, by which the base is a species able to donate an electron pair and an acid is a specie able to receive it ${ }^{7,23}$ can be equivalently applied to this system. Both approaches lead to Equation 17 as the representation of the process, in a single-step reaction. The presence of crystallization water was not considered, since the salt hydration degree varies with the temperature and with the crystallization conditions ${ }^{7,23}$.

$$
\mathrm{CaO}+\mathrm{SiO}_{2} \rightarrow \mathrm{CaSiO}_{3}
$$

According to Equation 17, the calcium oxide concentration reduces during the reaction, so it is expected that the medium $\mathrm{pH}$ reduces while the reaction proceeds, and then remains approximately constant when the limiting reactant is completely consumed.

In order to evaluate the consistency of this hypothesis, it is important to perform the integral analysis of the kinetic data. As extensively described by Levenspiel ${ }^{8}$, the integral method consists of integrating the kinetic expression proposed for a particular mechanism, and then comparing this theoretical curve of concentration varying with time with the experimental values.

If the proposed mechanism is correct, then there must be a satisfactory correspondence between the integral curve and the data obtained. Since the integral analysis is based on theoretical principles, the result provides strong evidence for supporting a particular reaction mechanism ${ }^{8}$.

Defining the chemical species $A$ as $\mathrm{CaO}$, species $B$ as $\mathrm{SiO}_{2}$, and the ratio $M$ as the relation between the initial concentration of $\mathrm{CaO}$ to the initial concentration of $\mathrm{SiO}_{2}$, it can be demonstrated that, if Equation 17 is correct, then the instant concentration of $\mathrm{CaO}$ is described by Equation $18^{8}$.

$$
F(t)=\ln \frac{M-x_{A}}{M\left(1-x_{A}\right)}=C_{A O}(M-1) k t
$$

Equation 18 applicability is limited to the situations where $M \neq 1$ and gives a linear plot when its left side, $\ln [(M-$ $\left.\left.x_{A}\right) / M\left(1-x_{A}\right)\right]$, is graphically correlated with the reaction time. If the experimental data is satisfactorily fitted by the straight line described by Equation 18, the fitting angular coefficient is equals to $C_{A 0}(M-1) k$. The adjusted curve usually shows a residual linear coefficient which is not related to the particular chemical kinetics but is due to random experimental errors ${ }^{8}$.

When using the methodology for the special case where $M=1$, the expression which must be valid if Equation 17 is correct is the Equation 19. This expression gives a linear plot when its left side is graphically correlated with reaction time. Now, the angular coefficient is equals to $k$, and the linear coefficient is equals to $1 / C_{A 0}{ }^{8}$.

$$
\frac{1}{C_{A}}=\frac{1}{C_{A O}}+k t
$$

Since there is significant difference depending on the value of $M$, experiments should be performed under conditions in which $M=1$, or in which $M$ is significantly different from $1^{8}$. Then, the experiments in this study were performed under conditions in which $M \approx 0.25$.

The experiments are summarized in Table 2. Calcium oxide was chosen as the limiting reactant, since this would provide the highest variation in $\mathrm{pH}$, enhancing the sensor sensitivity and allowing the correct detection of the reaction dynamics. Silica masses values were chosen in accordance with the following criteria: (1) the silica masses should be in large excess, but the value of $M$ should be maintained constant, for providing a simpler integral analysis; (2) the masses or proportions of reactants should be in accordance to the values used in studies applying Chapelle test; (3) since one of the objectives of this research was to provide savings of energy and use of chemical reactants, the values should be as lower as possible, but sufficiently large for maintaining the sensor capability in detecting $\mathrm{pH}$ variations during the reactions.

\section{Results and Discussion}

\subsection{Materials characterization}

The three silica materials were analyzed by SEM technique in Brazilian Nanotechnology National Laboratory (LNNano) microscope (FEI Quanta 650 FEG, USA), and the particles dimensions were graphically evaluated using the Image Viewer application of MATLAB 2016 (Mathworks, USA), an image processing routine that is capable of performing the quantification and measurement of different objects and shapes in a particular image. The SEM samples were prepared by depositing the powder over a conductive carbon 
Table 2. Kinetic experiments performed.

\begin{tabular}{|c|c|c|c|c|}
\hline Experiment & $\mathrm{SiO}_{2}$ source & $\begin{array}{c}\text { Temperature } \\
\left({ }^{\circ} \mathrm{C}\right)\end{array}$ & $\begin{array}{l}\text { Mass of } \\
\mathrm{CaO}(\mathrm{g})\end{array}$ & $\begin{array}{l}\text { Mass of } \\
\mathrm{SiO}_{2}(\mathrm{~g})\end{array}$ \\
\hline Q1 & $\begin{array}{l}\text { Crystalline } \\
\text { quartz }\end{array}$ & 65 & 0.7168 & 3.0500 \\
\hline Q2 & $\begin{array}{l}\text { Crystalline } \\
\text { quartz }\end{array}$ & 75 & 0.6635 & 3.0010 \\
\hline Q3 & $\begin{array}{l}\text { Crystalline } \\
\text { quartz }\end{array}$ & 55 & 0.7355 & 2.2257 \\
\hline Q4 & $\begin{array}{l}\text { Crystalline } \\
\text { quartz }\end{array}$ & 80 & 0.7267 & 2.9967 \\
\hline Q5 & $\begin{array}{l}\text { Crystalline } \\
\text { quartz }\end{array}$ & 70 & 0.7018 & 3.0047 \\
\hline Q6 & $\begin{array}{l}\text { Crystalline } \\
\text { quartz }\end{array}$ & 70 & 0.7054 & 3.7558 \\
\hline NS1 & Soot & 70 & 0.7047 & 2.9901 \\
\hline NS2 & Soot & 60 & 0.6153 & 2.9956 \\
\hline NS3 & Soot & 65 & 0.7149 & 3.0183 \\
\hline NS4 & Soot & 75 & 0.7149 & 3.0053 \\
\hline AS1 & $\begin{array}{l}\text { Active } \\
\text { silica }\end{array}$ & 75 & 0.7032 & 3.0019 \\
\hline AS2 & $\begin{array}{l}\text { Active } \\
\text { silica }\end{array}$ & 65 & 0.6373 & 3.0172 \\
\hline AS3 & $\begin{array}{l}\text { Active } \\
\text { silica }\end{array}$ & 60 & 0.7105 & 2.9907 \\
\hline AS4 & $\begin{array}{l}\text { Active } \\
\text { silica }\end{array}$ & 70 & 0.7042 & 3.0069 \\
\hline AS5 & $\begin{array}{l}\text { Active } \\
\text { silica }\end{array}$ & 80 & 0.7031 & 3.0007 \\
\hline AS6 & $\begin{array}{l}\text { Active } \\
\text { silica }\end{array}$ & 70 & 0.7054 & 2.9985 \\
\hline AS7 & $\begin{array}{l}\text { Active } \\
\text { silica }\end{array}$ & 75 & 0.7213 & 3.7277 \\
\hline
\end{tabular}

double-sided tape fixed in the stub. The samples were also carbon coated using a Bal-Tec SCD 050 Sputter Coater to obtain images under a high vacuum regime.

The XRD analyses were performed using a Rigaku DMAX 2200 diffractometer (Rigaku, Japan), with fine focus Cu tube $(\lambda=1.54 \AA)$, Bragg-Bretano geometry $(\theta-2 \theta), 0.02^{\circ}$ step and $2.5 \mathrm{~s} /$ step exposition time.

The quartz particles, shown in Figure 4, presented nonspherical morphologies, with considerable dispersion in their average dimensions (Table 3), which was expected due to the comminution process ${ }^{21,24,25}$.

According to the quartz XRD pattern, shown in Figure 5, the sample is comprised of practically $\alpha$-quartz, with the absence of amorphous halo. This same figure also shows that active silica and soot are essentially amorphous, with a low-intensity crystalline peak near $2 \theta=20^{\circ}$.

The SEM images obtained for the soot and for the active silica are shown in Figure 6 and Figure 7, respectively, and indicate spherical particles with diameters in the range of nanometers (Table 3). Active silica particles also showed a broader diameter

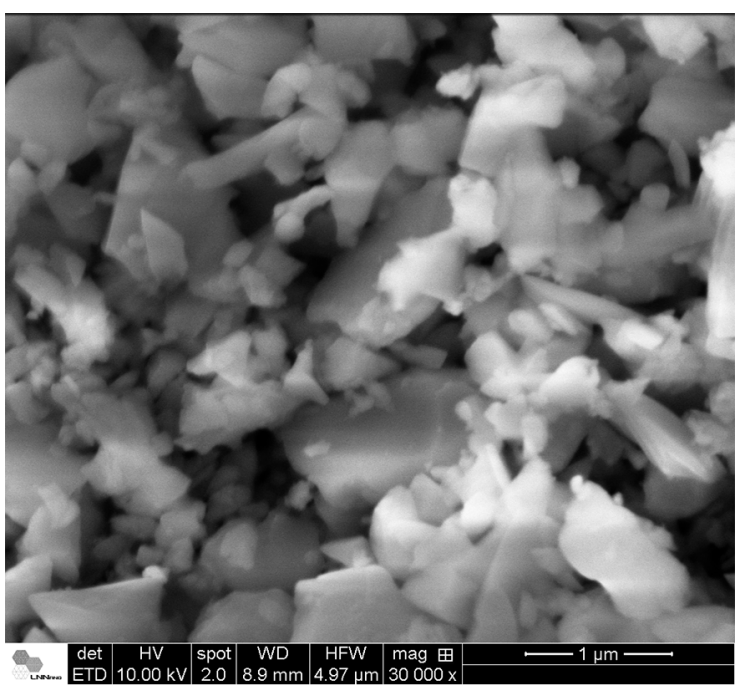

Figure 4. SEM image obtained for crystalline quartz.

Table 3. Average diameters and sphericities of analyzed silica particles.

\begin{tabular}{lcc}
\hline Material & $\begin{array}{c}\text { Average particle } \\
\text { diameter }\end{array}$ & Average sphericity \\
\hline Crystalline quartz & $(0.66 \pm 0.05) \mu \mathrm{m}$ & $0.68 \pm 0.03$ \\
Soot & $(110 \pm 5) \mathrm{nm}$ & $\approx 1$ \\
Active silica & $(243 \pm 7) \mathrm{nm}$ & $\approx 1$ \\
\hline
\end{tabular}

distribution in comparison to the soot, what is expected from the fact that the VAD process is capable of providing a finer control regarding the morphology of produced nanoparticles ${ }^{18}$.

\subsection{Kinetic experiments}

The experimental results are summarized in Table 4, which presents the reaction time, initial $\mathrm{pH}$, the total $\mathrm{pH}$ variation observed, the total conversion of calcium oxide and the linear correlation coefficient, $\mathrm{R}^{2}$, obtained for the application of Equation 18.

It is important to note that, after the mixture of the reactants, the system can experience a "dead-time", when no reaction is noticeable. This time must not be considered for the linear plot analyses, since the integral method is based on the assumption that the chemical reaction starts as soon as the reagents are put in contact $^{8}$.

From Figures 8 to 10 , it is possible to notice that the concentration of calcium oxide reaches a constant value in a relatively short time interval. In most of the reactions, the chemical process is not able to convert all of the $\mathrm{CaO}$, and the medium is still alkaline after the end of the reaction. It proves that not all of the silica is in acidic form, being not able to react with the base dissolved in liquid medium.

The graphics show a classical pattern obtained when a chemical equilibrium is reached ${ }^{9}$. This fact is in accordance with the silica chemical properties, since the acidic characteristics of the silica are related to its superficial free silanol concentration ${ }^{26,27}$, and the formation and consequently 


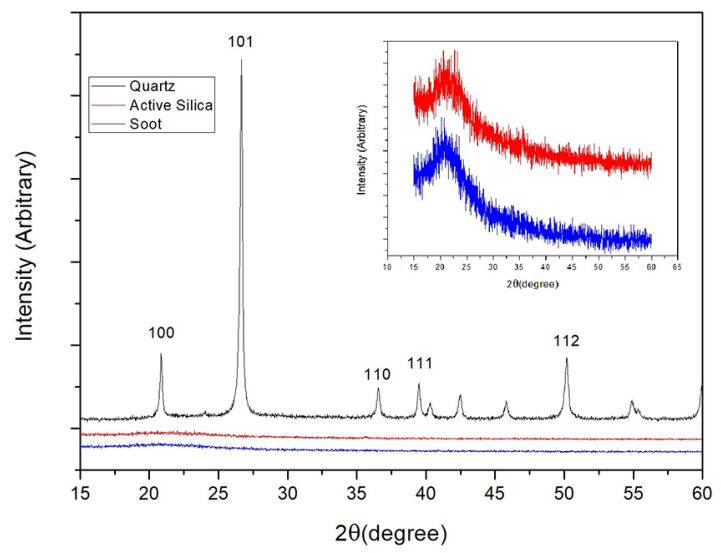

Figure 5. XRD pattern obtained for submicron quartz. The inset shows the soot and the active silica low-intensity crystalline peaks.

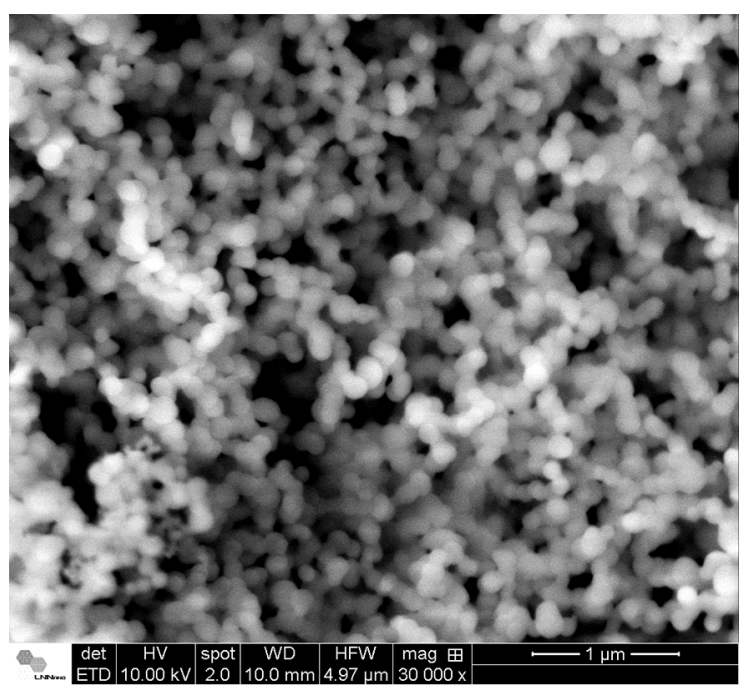

Figure 6. SEM image obtained for soot particles.

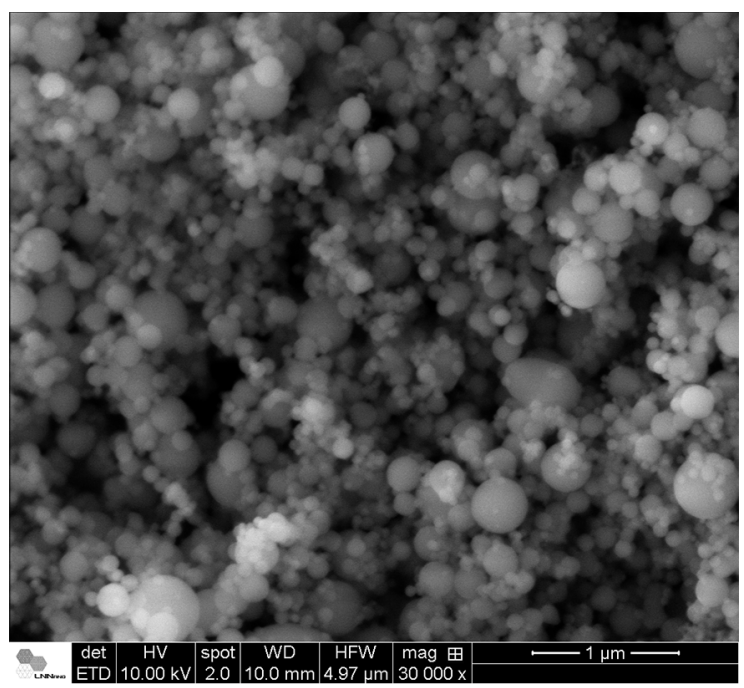

Figure 7. SEM image obtained for active silica particles.
Table 4. Summary of results obtained.

\begin{tabular}{lccccc}
\hline & $\begin{array}{c}\text { Reaction } \\
\text { time } \\
(\mathrm{min})\end{array}$ & & $\begin{array}{c}\text { Total pH } \\
\text { variation } \\
\text { observed }\end{array}$ & $\begin{array}{c}\text { Total } \\
\text { conversion } \\
\text { of CaO } \\
(\%)\end{array}$ & $\begin{array}{c}\text { R }{ }^{2} \text { obtained } \\
\text { for linear } \\
\text { adjust }\end{array}$ \\
\hline Q1 & 220 & 11.657 & 1.179 & 99.7 & 0.9825 \\
Q2 & 150 & 11.233 & 0.563 & 32.7 & 0.9615 \\
Q3 & 130 & 12.005 & 0.395 & 100.0 & 0.9246 \\
Q4 & 230 & 11.267 & 0.903 & 39.8 & 0.9422 \\
Q5 & 200 & 11.333 & 0.510 & 54.1 & 0.9047 \\
Q6 & 240 & 11.484 & 2.169 & 74.0 & 0.9746 \\
NS1 & 30 & 11.957 & 0.936 & 100.0 & 0.8467 \\
NS2 & 30 & 11.548 & 0.539 & 100.0 & 0.9088 \\
NS3 & 120 & 11.100 & 0.296 & 31.2 & 0.9301 \\
NS4 & 100 & 10.852 & 0.997 & 13.9 & 0.9048 \\
AS1 & 80 & 11.546 & 2.072 & 81.8 & 0.9814 \\
AS2 & 80 & 11.373 & 1.725 & 60.5 & 0.9799 \\
AS3 & 50 & 11.817 & 2.142 & 100.0 & 0.9919 \\
AS4 & 10 & 12.045 & 2.305 & 100.0 & - \\
AS5 & 50 & 11.430 & 2.152 & 62.5 & 0.8992 \\
AS6 & 80 & 11.436 & 2.109 & 66.6 & 0.9036 \\
AS7 & 80 & 11.220 & 1.978 & 40.4 & 0.8972 \\
\hline & & & & &
\end{tabular}

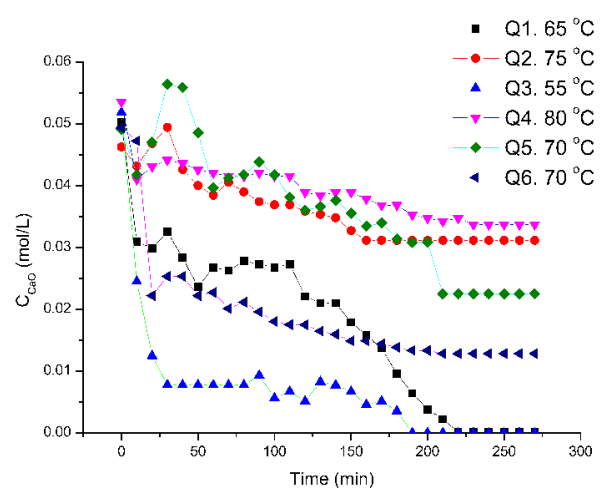

Figure 8. Kinetic experiments conducted with crystalline quartz as the silica source. The lines are guides for the eye

reactivity of this group is represented by a chemical equilibrium between silica and water ${ }^{26,27}$. The consequence is that, depending on the particular conditions of the medium, not all of the silanols are able to react as acid groups, and such acid groups are in equilibrium with neutral groups ${ }^{26,27}$.

It is important to realize that, when the reaction is complete, the system $\mathrm{pH}$ is considerably higher than 8 , which is the approximate turning point of the phenolphthalein indicator at $25^{\circ} \mathrm{C}^{10}$. It can be expected errors in the results for titrations if they are conducted at the same temperature of the reaction systems, higher than $25^{\circ} \mathrm{C}$, and it is also important to analyze 


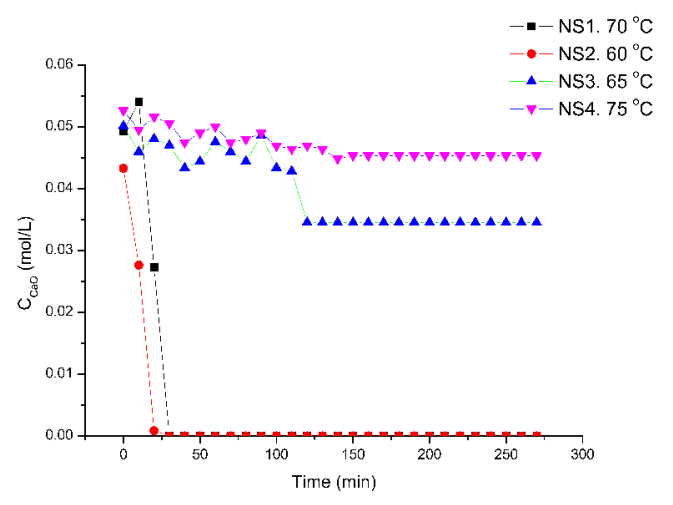

Figure 9. Kinetic experiments conducted with soot as the silica source. The lines are guides for the eye.

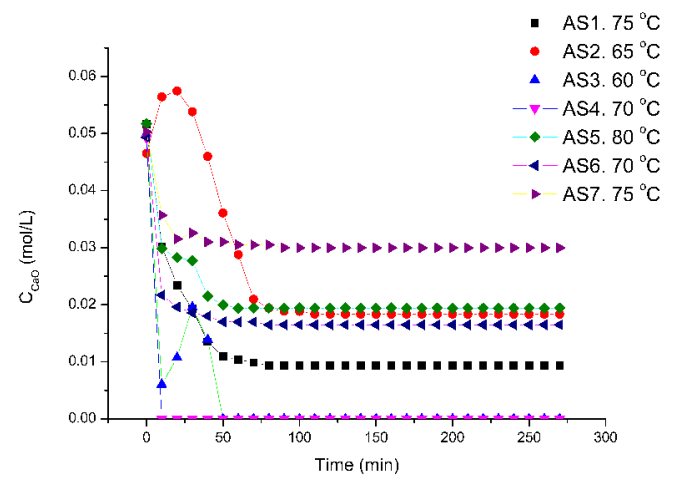

Figure 10. Kinetic experiments conducted with active silica as the silica source. The lines are guides for the eye.

if the equilibrium between the two different ionization states of the indicator ${ }^{10}$ is substantially affected by the temperature.

This variation would change the turning point, and a complete thermodynamic analysis would be necessary to verify if the equivalence point coincides with the indicator turning point ${ }^{10}$. Even if the titration temperature is rigidly controlled under $25{ }^{\circ} \mathrm{C}$, the reversibility of the calcium oxide reaction can disturb the analysis since, according to Equations 3, 4 and 7, the species concentration in equilibrium can change with the variation of the temperature $e^{8,9}$.

The solutions for Equations 3, 4 and 7 also show that, for temperatures higher than $55^{\circ} \mathrm{C}$, the neutral concentration of hydroxide ions is at least one order of magnitude higher than for $25^{\circ} \mathrm{C}$. The conclusion is that the neutral $\mathrm{pH}$, where hydrogen and hydroxide ions concentrations are equal, is considerably higher than 7. As the Chapelle test does not consider this factor ${ }^{4,6}$, one can expect several deviations between the "reactivity" calculated by this test and the real value of the reaction yield.

An accurate analysis of the results of the Chapelle test is difficult, since the medium temperature is increased until the aqueous solution reaches its bubble point ${ }^{4,6}$. As the bubble point is a strong function of the local atmospheric pressure, and of the particular chemical species in the medium and their concentrations ${ }^{9}$, there is no standardization in the test temperature and, depending on all of these conditions, very specific reaction rates and equilibrium states will be obtained for the reaction ${ }^{8,9}$. Since such factors change the observed yield, it will not be possible to compare results from different Chapelle test experiments, even when real experimental temperatures are documented.

The reaction times given in Table 4 are the time differences between the first instant when the concentration shows variation and the instant when the final plateau is reached, evidencing the end of the reaction. For each one of the three different silica sources, the mean values of the reaction times were evaluated with the ANOVA ("analysis of variance"). It is a statistical procedure for evaluating the confidence intervals of the mean results obtained for different samples and the significance of the differences between different means. If the confidence intervals show no intersections, it is possible to conclude with a given confidence - in this research, 95\% confidence - that the mean results are statistically different.

Figure 11 shows the $95 \%$ confidence intervals of the mean reaction times observed for the crystalline quartz tests, for the soot tests, and for the active silica tests, obtained with the ANOVA test performed with $95 \%$ confidence, and Table 5 summarizes the values of the mean reaction times and the calculated confidence intervals. The analysis was performed in Minitab 17 (Minitab Inc, USA) and indicates a significant difference in the mean reaction time observed for crystalline quartz, which is considerably higher than the observed for the other silica sources, and no significant difference between the mean reaction times observed for active silica and for soot tests.

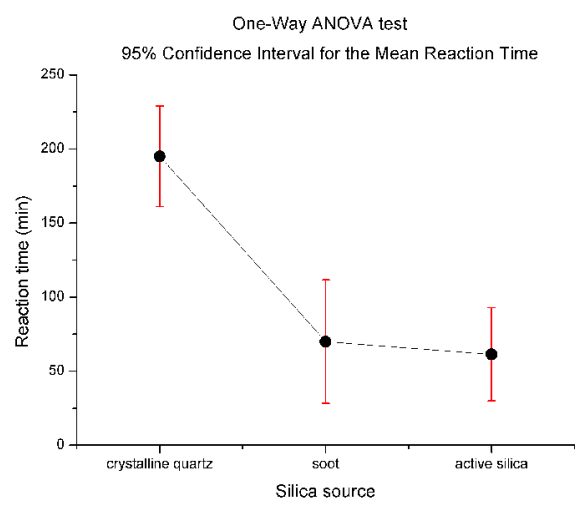

Figure 11. ANOVA test of the reaction times, with $95 \%$ confidence interval (CI) for mean reaction time. The lines connecting dots and the horizontal lines are guides for the eye.

In all of the experiments shown in Figure 8, there is an initial baseline before calcium oxide concentration varies significantly, and it is a determinant factor for the higher reaction time. As the silica amorphous materials have a lower 
Table 5. Mean reaction times and $95 \%$ confidence intervals calculated.

\begin{tabular}{lcc}
\hline Silica source & $\begin{array}{c}\text { Mean reaction } \\
\text { time }(\mathrm{min})\end{array}$ & $\begin{array}{c}95 \% \text { confidence } \\
\text { interval for mean } \\
\text { reaction time }(\mathrm{min})\end{array}$ \\
\hline Crystalline quartz & 195.0 & $(161.1 ; 228.9)$ \\
Soot & 70.0 & $(28.4 ; 111.6)$ \\
Active silica & 61.4 & $(30.0 ; 92.9)$ \\
\hline
\end{tabular}

reaction time, a feasible hypothesis is that the crystalline structure is responsible for the lower reaction rate.

It is possible that, before it is able to react with the free basic species dissolved in the liquid medium, the silica silanol groups must be exposed in the surface, and the rigidity of the crystalline structure reduces the number of available groups $^{26,27}$. Then, the initial baseline would be caused by the combined effect of the temperature, the electric interaction between the silica surface and the hydroxide negatively charged ions, and the liquid fluid dynamics.

These effects would be responsible for inducing the presence of silanol groups in silica surface, in accordance with the Zhuravlev model for silica chemistry ${ }^{27}$. Then, the effective reaction only starts when there are sufficient acid groups able to neutralize the base.

Another important possibility is the effect of the surface area - it is a general principle in chemical kinetics that the reaction rate rises with the increase in surface area ${ }^{8}$.

Figures 4, 6 and 7 and the data presented in Table 3 evidence that the crystalline quartz shows a higher value of particle mean diameter, a lower value of sphericity and also has a lower specific surface area, what decreases the interaction area between the silica particles and the $\mathrm{CaO}$, reducing the rate of reaction, according to the Langmuir theory ${ }^{8}$. The quantification of the adsorption sites on silica surface and the specific area of the particles needs a specific BET study ${ }^{8}$, and the analysis of this effect is an important limitation of the presented methodology. With the proposed instrumentation, it can only be performed qualitatively when there are available SEM images which evidence the differences in the specific area.

In most of the experiments conducted with soot and active silica, the reaction reaches the equilibrium plateau in a very short time, making it difficult to compare the results for different temperatures, since the temperature effect has little significance in these cases.

However, in quartz experiments, in which the time scale is wider, the tendency observed is in accordance with the hypothesis made. The initial reaction rate is increased with temperature, and both the Equation 17 and the analysis of Lux-Flood and Lewis theories suggest an acid-base neutralization reaction.

It is commonly observed that acid-base reactions are considerable exothermic ${ }^{7-10}$, and the chemical equilibrium in reactions with such thermodynamic behavior is favored by lower temperatures ${ }^{8,9}$.
Figure 8 shows the correspondence between the total $\mathrm{CaO}$ converted and this hypothesized temperature behavior: the two lowest temperatures, 65 and $55^{\circ} \mathrm{C}$, show complete conversion; the two experiments conducted at $70{ }^{\circ} \mathrm{C}$ show a small variation, expected due to stochastic effects, and both cases show higher conversions than the other two experiments conducted in higher temperatures. The experiment performed at $75^{\circ} \mathrm{C}$ exhibits a lower conversion than the experiments conducted at $70^{\circ} \mathrm{C}$, but a higher conversion than the experiment carried out at $80^{\circ} \mathrm{C}$. Finally, the experiment conducted at $80^{\circ} \mathrm{C}$, the highest temperature tested, shows the lowest total conversion of $\mathrm{CaO}$.

Table 6 summarizes a comparison between the analyses performed with the methodology proposed in this research and the Chapelle test. It can be easily concluded that there are advantages related to time, energy consumed, and to more accuracy and confidence obtained in the experiments.

\subsection{Mechanistic integral analysis}

The fitting of the experimental values to the straight line described by Equation 18 was applied in order to verify if the kinetic data obtained was consistent with the proposed chemical mechanism. The linear correlation coefficient obtained for the adjusted straight lines were $\mathrm{R}^{2} \cong 1$ for all of the experiments, and this fact reveals a strong linear correlation and consistency with the hypothesis. It is worth noticing that the coefficient could not be calculated for experiment AS4 due to the low reaction time, since it is necessary to remove both the dead-time and the plateau, as it was already mentioned.

Figure 12 shows the straight lines $F(t)$, defined by Equation 18, obtained for 4 experiments using crystalline quartz, and Figure 13 shows the straight lines obtained for 1 experiment using soot and for 2 experiments using active silica as the silica source.

The data show a complete agreement with the expected behavior, and it is possible to observe that some experiments for active silica obtained $\mathrm{R}^{2}>0.98$, what is technically a perfect straight line. The deviations from the linear regression can be attributed to stochastic errors during the experiments.

The fitted straight lines $F(t)$ obtained can be described by their angular and linear coefficients, and the angular coefficient is related to the kinetic rate constant $k$ in each experimental temperature accordingly to Equation 18. Table 7 summarizes the coefficients obtained for each one of the linear adjustments, even the ones that were not shown in Figures 12 and 13, but the analysis could not be performed for experiment AS4 due to the low number of data collected. The main result showed in Table 7 is the consistency of the data, that show similar values and order of magnitudes for the same parameters. This data is important for reinforcing the kinetic hypothesis, a 1:1 reaction.

It is not possible to observe neither clear differences in the mean values of the parameters nor a tendency of the kinetic constants with the increase in the temperature range tested but, as 
Table 6. Comparison between the proposed methodology and the Chapelle test $\mathrm{t}^{4-6}$.

\section{Kinetic and thermodynamic analysis proposed}

The analysis takes less than 3 hours.
Chapelle test

The analysis takes 16 hours plus the time needed for the titration of the sample.

Energy and time savings, due to the use of less time and lower temperatures.

Procedure with more accuracy, more confidence, and more robustness, due to the use of electronic, simple and non-expensive equipment, without errors associated with visual procedures.

The experimenter needs to heat the aqueous medium until its bubble point and needs to maintain this temperature for 16 hours.

Lack of robustness to experimental errors. Sensitivity to visual errors, due to the use of colorimetric indicators in a turbid medium, and sensitivity to errors due to analyst's lack of experience in performing the titration.

The analysis does not take in account neither the temperature and pressure effects nor the effect of the differences in suspensions The analysis takes in account the composition of the medium and composition; such differences can change both the titration results the temperature and pressure effects on the ionic equilibrium of and the bubble point temperature. Different Chapelle experiments water, allowing the comparison between experiments performed cannot be compared, since the environmental temperatures are at different temperatures and different compositions of the medium. different. Chapelle tests are always performed with the same proportions, so the effect of the composition cannot be evaluated and chemical reactants cannot be saved.

Allows the observation of the presence of chemical equilibrium and Does not allow the observation of the dynamic behaviors, since of the entire kinetic behavior.

The analysis is done in situ, and all environmental effects are taken in account. There is no risk of disturbance in the chemical equilibrium due to the analysis procedure.

The procedure is fast, so the effects of parallel reactions with gases in atmospheric air are insignificant.

Kinetic experiments allow the quantification of interference caused by agents like: agitation power, solid-liquid equilibrium effects, reaction limitations by an increase in mass transfer resistance, and so on.

The sample must be carefully cooled to $25{ }^{\circ} \mathrm{C}$ for the titration procedure, due to the colorimetric agent equilibrium. This cooling process can change the apparent conversion, since the reversible reaction yield is dependent on the temperature.

The experiment takes long time, 16 hours, and parallel reactions, such as the $\mathrm{CaO}$ neutralization by the air $\mathrm{CO}_{2}$, can affect significantly the result.

The analysis does not allow the evaluation of the interference caused by external effects, and all of the environmental parameters must be carefully controlled and kept constant.

Chapelle test does not provide information about the intrinsic kinetic mechanism, providing no information about the phenomena involved.

The methodology provides information on the intrinsic kinetic mechanisms, helping the elucidation of the phenomenon.

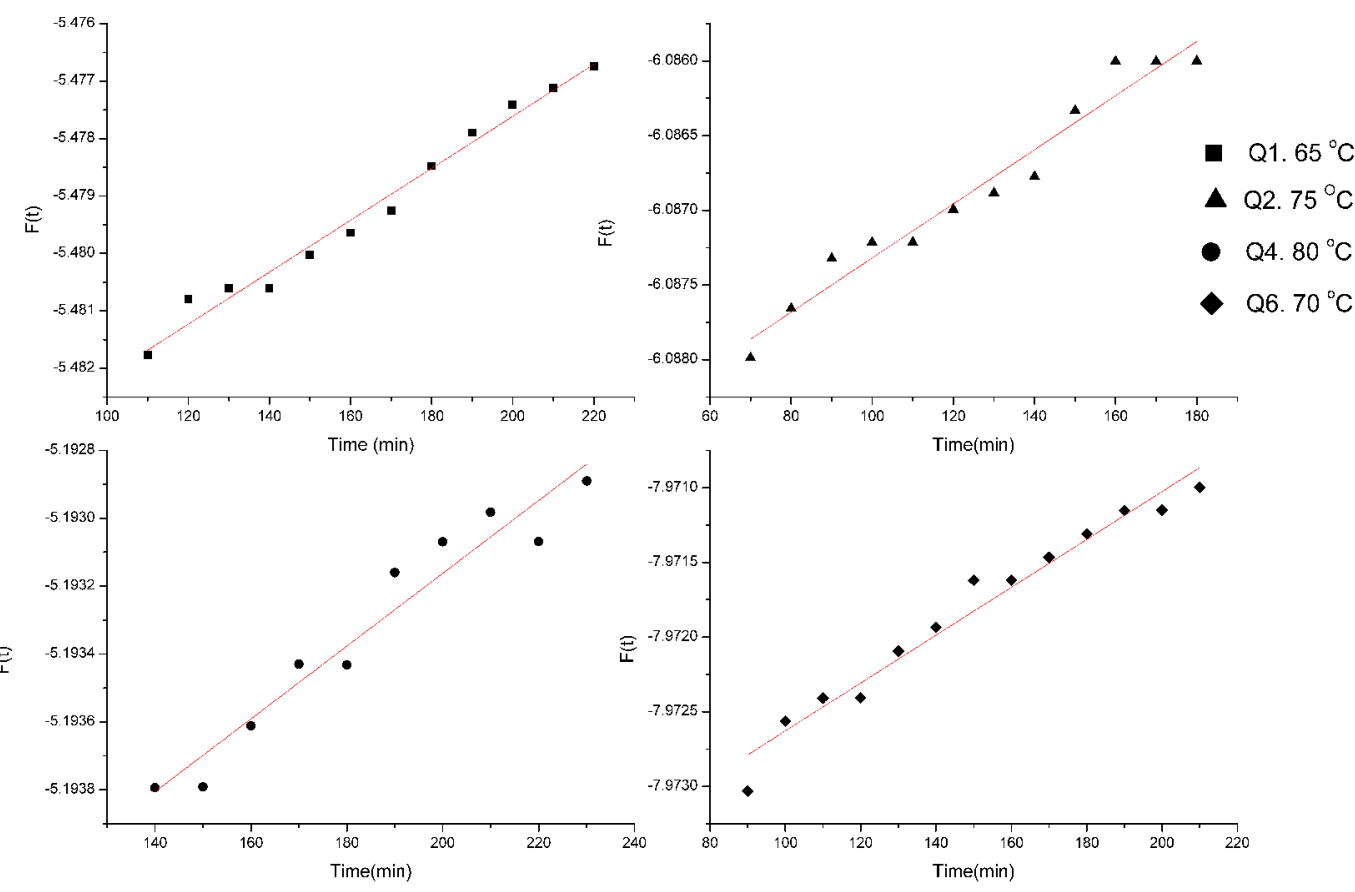

Figure 12. Straight lines obtained for four of the experiments using crystalline quartz, in the integral analysis. 


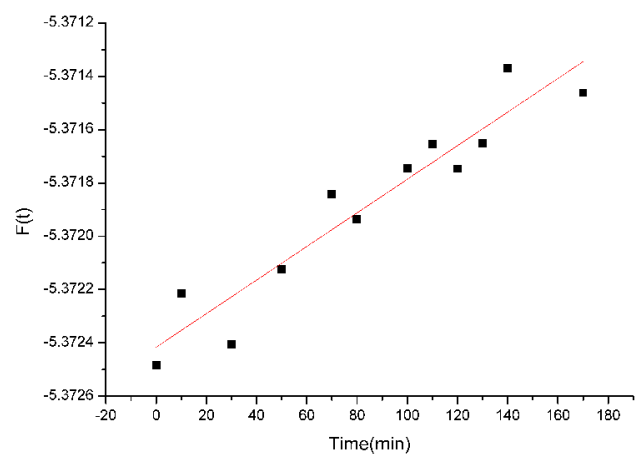

- NS4. $75^{\circ} \mathrm{C}$

$\nabla$ AS $1.75^{\circ} \mathrm{C}$

- AS2. $65{ }^{\circ} \mathrm{C}$
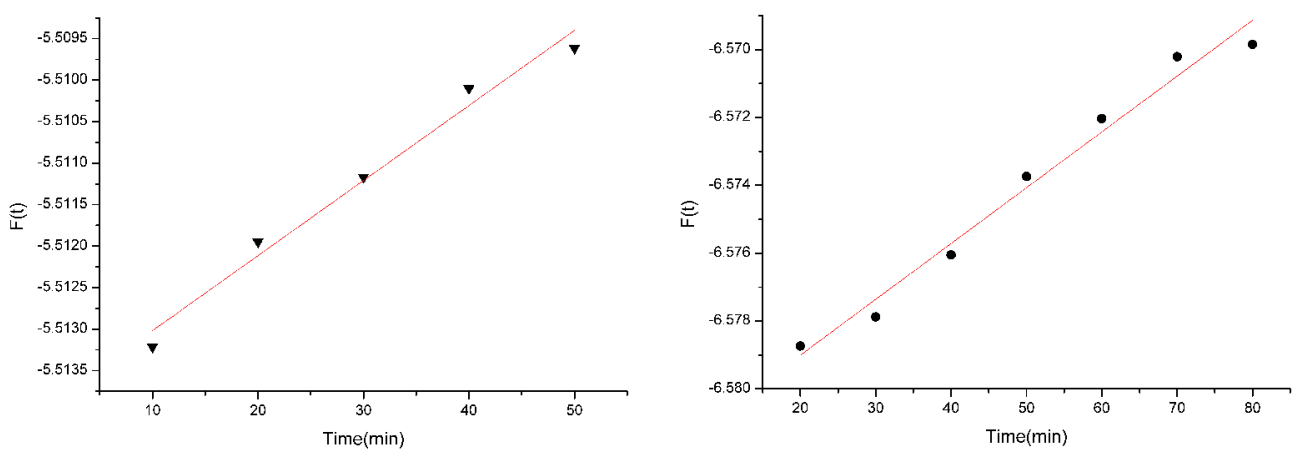

Figure 13. Straight lines obtained for one experiment using soot and for two experiments using active silica, in the integral analysis.

Table 7. Angular and linear coefficients of Equation 18 and numerical values of the rate constants obtained for each experiment.

\begin{tabular}{lccccc}
\hline Experiment & Temperature $\left({ }^{\circ} \mathrm{C}\right)$ & $\begin{array}{c}\text { Angular coefficient } \\
\text { of the linear } \\
\text { regression }\end{array}$ & $\begin{array}{c}\text { Linear coefficient } \\
\text { of the linear } \\
\text { regression }\end{array}$ & $\begin{array}{c}\text { Numerical value of } \\
\text { kinetic rate constant } \\
\boldsymbol{k} \text { obtained }\end{array}$ & $\begin{array}{c}\mathrm{R}^{2} \text { obtained for } \\
\text { linear adjust }\end{array}$ \\
\hline Q1 & 65 & $4.52 \times 10^{-5}$ & -5.49 & $1.20 \times 10^{-3}$ & 0.9825 \\
Q2 & 75 & $1.81 \times 10^{-5}$ & -6.09 & $5.13 \times 10^{-4}$ & 0.9615 \\
Q3 & 55 & $8.74 \times 10^{-6}$ & -2.93 & $2.61 \times 10^{-4}$ & 0.9246 \\
Q4 & 80 & $1.07 \times 10^{-5}$ & -5.20 & $2.70 \times 10^{-4}$ & 0.9422 \\
Q5 & 70 & $2.13 \times 10^{-5}$ & -5.55 & $5.79 \times 10^{-4}$ & 0.9047 \\
Q6 & 70 & $1.49 \times 10^{-5}$ & -7.97 & $3.78 \times 10^{-4}$ & 0.9746 \\
NS1 & 70 & $3.27 \times 10^{-4}$ & -5.46 & $8.88 \times 10^{-3}$ & 0.8467 \\
NS2 & 60 & $3.93 \times 10^{-4}$ & -6.89 & $1.16 \times 10^{-2}$ & 0.9088 \\
NS3 & 65 & $1.64 \times 10^{-5}$ & -5.41 & $4.39 \times 10^{-3}$ & 0.9301 \\
NS4 & 75 & $6.30 \times 10^{-6}$ & -5.37 & $1.61 \times 10^{-4}$ & 0.9048 \\
AS1 & 75 & $9.05 \times 10^{-5}$ & -5.51 & $2.34 \times 10^{-3}$ & 0.9814 \\
AS2 & 65 & $1.64 \times 10^{-4}$ & -6.58 & $4.56 \times 10^{-3}$ & 0.9799 \\
AS3 & 60 & $1.79 \times 10^{-4}$ & -5.38 & $4.80 \times 10^{-3}$ & 0.9919 \\
AS4 & 70 & - & - & $-5.28 \times 10^{-3}$ & - \\
AS5 & 80 & $4.96 \times 10^{-5}$ & -5.51 & $-55 \times 10^{-4}$ & 0.8992 \\
AS6 & 70 & $1.68 \times 10^{-5}$ & -5.47 & -7.60 & 0.9036 \\
AS7 & 75 & $2.95 \times 10^{-5}$ & & 0.8972 \\
\hline
\end{tabular}


it was already cited, a complete study of the correlation between kinetics and the specific surface area should be performed in future works in order for determining possible correlations.

\section{Conclusions}

The integral analysis provided semi-empirical evidence for the proposed mechanism, a 1:1 reaction, since it is very unusual for a chemical reaction to agree with more than one integral straight line ${ }^{8}$, and the satisfactory adjustment to the proposed model was obtained for 16 experiments performed under different conditions. It is important, however, to emphasize that, due to the very small scales of dimensions and time events, it is very difficult to prove that a particular mechanism is valid for generalized cases, since the events take place in the molecular and atomic levels ${ }^{8}$. A proposed mechanism can be accepted as a right and consistent one until unquestionable facts against it have been discovered ${ }^{8}$.

The statistically significant difference of mean reaction times obtained for different silica sources provided evidence that the crystalline structure and the particles specific surface area can be determinant factors for the reaction rates, and these hypotheses are supported by the silica model developed by Zhuravlev $^{27}$ for the chemistry of silanol groups in water, and by the Langmuir kinetic model ${ }^{8}$, respectively. It was verified a reaction time of $195 \mathrm{~min}$ for crystalline silica particles with average diameter of $0.66 \mu \mathrm{m}$, and reaction time of $61 \mathrm{~min}$ for active silica particles with average diameter of $243 \mathrm{~nm}$. Both reaction times are very lower than the standard time of the Chapelle test, 16 hours plus the time required for the titration chemical analysis.

It is important to realize that the proposed analysis has many advantages in comparison to the traditional Chapelle test. Besides the less time and energy-consuming, the procedure prevents the analysis to be affected by parallel reactions, such as the reaction with carbon dioxide from atmospheric air, and takes in account the effects of the temperature, allowing the comparison between experiments performed in very different conditions, since the reaction parameters are corrected by rigorous thermodynamic equations. It is also able to eliminate the imprecisions related to the visual titration technique, that can show unsatisfactory results in excessive turbid media, and the fluctuations related to the poor temperature control. It is possible to evaluate the temperature effects on the reversibility of the reaction as well, but there is an intrinsic limitation in evaluating the effect of the particles surface area, which needs a separate BET study.

The next studies should then evaluate the specific surface area effect on chemical reaction rate and evaluate if temperatures even lower than $55^{\circ} \mathrm{C}$ can be employed for the analysis, depending on the granulometry, providing even more energy savings and helping the elucidation of the effects of the surface energy on interactions between nanoparticles.

The titration with chemical indicators which change their colors in the turning point - used in Chapelle test - is a classic analytical procedure, but it is particularly sensitive to the analyst experience and familiarity with the technique ${ }^{10}$. In this sense, the use of an electronic, simple and non-expensive device, such as the $\mathrm{pH}$ meter, provides more accuracy and confidence to the results obtained and important time savings, being more robust in relation to the possible errors of the experimenters.

\section{Acknowledgements}

The authors would like to thank Brazilian Nanotechnology National Laboratory LNNano) for the use of the facilities, CNPq, CAPES, and FAPESP Grants 2015/02185-3 and 2017/20445-8 for financial support.

\section{References}

1. Poon CS, Lam L, Kou SC, Wong YL, Wong R. Rate of pozzolanic reaction of metakaolin in high-performance cement pastes. Cement and Concrete Research. 2001;31(9):1301-1306.

2. Caldarone MA, Gruber KA, Burg RG. High Reactivity Metakaolin (HRM): A New Generation Mineral Admixture for High Performance Concrete. Concrete International. 1994;16(11):37-40.

3. Zhang MH, Malhotra VM. Characteristics of a thermally activated alumino-silicate pozzolanic material and its use in concrete. Cement and Concrete Research. 1995;25(8):17131725 .

4. Perraki T, Kakali G, Kontoleon F. The effect of natural zeolites on the early hydration of Portland cement. Microporous and Mesoporous Materials. 2003;61(1-3):205-212.

5. Sabir BB, Wild S, Bai J. Metakaolin and calcined clays as pozzolans for concrete: a review. Cement and Concrete Composites. 2001;23(6):441-454.

6. Kakalia G, Perraki T, Tsivilis S, Badogiannis E. Thermal treatment of kaolin: the effect of mineralogy on the pozzolanic activity. Applied Clay Science. 2001;20(1-2):73-80.

7. Cotton FA, Wilkinson G. Advanced Inorganic Chemistry. Hoboken: John Wiley and Sons; 1980.

8. Levenspiel O. Chemical Reaction Engineering. Hoboken: John Wiley and Sons; 1999.

9. Smith JM, Van Ness HC, Abbott MM. Introduction to Chemical Engineering Thermodynamics. New York: McGraw-Hill; 2004.

10. Skoog DA, West DM, Holler FJ, Crouch SR. Fundamentals of Analytical Chemistry. Pacific Grove: Brooks Cole; 2013.

11. Olofsson G, Hepler LG. Thermodynamics of ionization of water over wide ranges of temperature and pressure. Journal of Solution Chemistry. 1974;4(2):127-143.

12. Lewis GN, Randall M. The thermodynamic treatment of concentrated solutions, and applications to thallium amalgams. Journal of the American Chemical Society. 1921;43(2):233-254. 
13. Edwards TJ, Newman J, Prausnitz JM. Thermodynamics of aqueous solutions containing volatile weak electrolytes. AIChE Journal. 1975;21(2):248-259.

14. Helgeson H, Kirkham D. Theoretical Prediction of the Thermodynamic Behavior of Aqueous Electrolytes at High Pressures and Temperatures: II. Debye-Hückel Parameters for Activity Coefficients and Relative Partial Molal Properties. American Journal of Science. 1974;274(10):1199-1261.

15. Çengel YA, Cimbala JM. Fluid Mechanics: Fundamentals and Applications. McGraw-Hill; 2006.

16. Uematsu M, Frank EU. Static Dielectric Constant of Water and Steam. Journal of Physical and Chemical Reference Data. 1980;9(4):1291-1306.

17. Bailey J, Ollis D. Biochemical Engineering Fundamentals. New York: McGraw-Hill; 1986.

18. Santos JS, Ono E, Fujiwara E, Manfrim TP, Suzuki CK. Control of optical properties of silica glass synthesized by VAD method for photonic components. Optical Materials.2011;33(12):1879-1883.

19. Manfrim TP, Ono E, Fujiwara E, Santos MFM, Boery MNO, Santos JS, et al. A method to synthesize $\mathrm{SiO}_{2}-\mathrm{TiO}_{2}$ glasses based on the synergy between VAD and ALD techniques: study of $\mathrm{TiO}_{2}$ doping profile along radial direction. Optical Materia ls.2011;33(12):1938-1942.
20. Fecht HJ, Hellstern E, Fu Z, Johnson WL. Nanocrystalline metals prepared by high-energy ball milling. Metallurgical Transactions A. 1990;21:2333-2337.

21. Sopicka-Lizer M, ed. High-Energy Ball Milling: Mechanochemical Processing of Nanopowders. Cambridge: Woodhead Publishing; 2010.

22. Fujiwara E, Schenkel EA, Santos MFM, Suzuki CK. Concentration measurements in silica and quartz nanofluids by optical fiber sensor. In: SPIE Proceedings 9634; $24^{\text {th }}$ International Conference on Optical Fibre Sensors; 2015 Sep 28-Oct 2; Curitiba, PR, Brazil.

23. Lee JD. Concise Inorganic Chemistry. Hoboken: WileyBlackwell; 1999.

24. Chen M, McCauley JW, Hemker KJ. Shock-induced localized amorphization in boron carbide. Science. 2003;299(5612):15631566.

25. Koch CC. Top-Down Synthesis of Nanostructured Materials: Mechanical and Thermal Processing Methods. Reviews on Advanced Materials Science. 2003;5(2):91-99.

26. Iler RK. The Chemistry of Silica Solubility, Polymerization, Colloid and Surface Properties, and Biochemistry. Hoboken: John Wiley and Sons; 1978.

27. Zhuravlev LT. The surface chemistry of amorphous silica. Zhuravlev model. Colloids and Surfaces A: Physicochemical and Engineering Aspects. 2000;173(1-3):1-38. 